


\section{Imaging techniques in lung cancer}

\section{Summary}

Conventional chest radiography, computed tomography (CT), magnetic resonance (MR) and positron emission tomography (PET) are all imaging techniques used for the detection, characterisation, staging and follow-up of lung cancer. The success of $C T$ is related to the fact that very detailed imaging information of the localisation and the extent of the tumour, the presence of enlarged lymph nodes and the presence of metastatic disease can be provided. Integrated PET/CT combines anatomical information from CT with metabolic information from PET. PET/CT is nowadays the best imaging technique for the staging of lung cancer. MR was used for a long time as a problemsolving tool; however, with the development of new application methods, MR becomes a promising imaging modality in the evaluation of lung cancer patients. In this review, the use of these imaging techniques in the evaluation of lung cancer will be discussed.

\section{Imaging techniques}

\section{Chest radiograph}

Chest radiography is the most commonly performed diagnostic imaging test for the diagnosis of many pulmonary diseases throughout the world. Simplicity, low cost, low radiation, large amounts of information and wide availability are many advantages of this technique [1]. The most satisfactory routine radiographic views are the posteroanterior (PA) and lateral projections with the patient standing up. Lordotic projections, with the X-ray beam in a cephaled angle of $15^{\circ}$, can be used for an improved visibility of the lung apices, superior mediastinum and thoracic inlet [2]. Oblique studies are sometimes useful in locating a pleural or chest wall disease process.

Many advances in conventional thoracic imaging are made, thanks to several technical innovations in the fields of digital detection and post-processing $[3,4]$. The most remarkable is the rapid conversion from film-based to digital radiographic (DR) systems [5, 6]. Advantages of DR systems are the high image quality and the potential for dose reduction together with a favourable cost-benefit ratio and an increased efficiency [7]. Post-processing techniques in digital radiography are temporal subtraction and dual-energy subtraction [8]. To increase the accuracy of detection of lung nodules, nowadays, temporal subtraction can be used [9]. Temporal subtraction is based on a subtraction of two postero-anterior chest radiographs taken at two different points in time [1]. These images can show subtle parenchymal changes that can be overlooked due to overlying and distracting anatomical structures. Dual-energy subtraction uses the different absorption characteristics of calcified and non-calcified structures as a function of exposure voltage. These images, obtained with high- and low-energy $X$-rays have been shown to also improve the detection of pulmonary nodules and may improve lung cancer screening using conventional X-rays $[10,11]$.

Chest radiography was the first imaging technique for lung cancer screening. Large randomised trials were performed in the USA and in Europe to investigate the role of chest radiography in lung cancer screening $[12,13]$. These studies found a higher incidence of
W. De Wever

J. Coolen

J.A. Verschakelen

Department of Radiology, University Hospitals Leuven, Herestraat 49, 3000 Leuven, Belgium

\section{Correspondence}

W. De Wever

Radiology Department

University Hospitals Leuven

Herestraat 49

3000 Leuven

Belgium

walter.dewever@uzleuven.be

Competing interests None declared. 
resectable diseases in the screened population, but none of them showed a lung cancer mortality reduction. Since these disappointing results, lung cancer screening with chest radiography has been abandoned.

Chest radiography can be used for characterisation of lung lesions as benign or malignant. The speed of growth of a lesion over time indicates the cell replication rate within the lesion and gives information about its benign or malignant character. Tumour doubling time is an independent and significant prognostic factor for lung cancer patients. SPRATT et al. [14] found a mean doubling time of 3.1 months for squamous cell carcinoma, 9 months for adenocarcinomas and 3 months for undifferentiated cancer on chest radiography.

The purpose of lung cancer staging is to determine the extent of disease in order to select patients who will benefit from surgery, and also to determine prognosis. For staging purposes, chest radiography can assess lesion size, and can demonstrate features such as postobstructive collapse, pleural effusion and, in some instances, extrapulmonary spread. However, chest radiography cannot detect invasion of the chest wall, diaphragm and mediastinum, and nodal involvement [15].

\section{Computed tomography}

Computed tomography (CT) is the second most important imaging modality of the chest. Before 1991, a conventional or incremental CT scan of the chest was performed and consisted of a series of cross-sectional slices obtained during suspended respiration (the "stop-and-go" method). In 1991, helical CT was introduced in the imaging of the chest and dramatically improved the quality of $C T$ images of thoracic structures [16]. Helical CT produces a single volumetric dataset within one breath-hold during continuous scanning while the patient is moved through the $C T$ gantry. The X-ray beam traces a helical or spiral curve in relation to the patient. More recently, multi-detector CT (MDCT) scanners have been developed $[16,17]$. These new CT scanners use multiple rows $(4,8,16,32,64$ or 128 rows) of detectors. The advantages of MDCT have revolutionised the diagnostic approach to lung cancer. First, there is an increased temporal resolution. Data acquisition is so rapid that scanning of the entire lung can be performed within a single breath-hold. Due to this better temporal resolution, there is also a better contrast material administration possible.
Secondly, there is an increased spatial resolution. Continuous acquisition of thin slices allows the improvement of the image quality of multiplanar reconstruction (MPR) images $[18,19]$. Twodimensional multiplanar reformation images are single-voxel-thick sections displayed in the coronal, sagittal or oblique plane. Curved reformations along the long axis of the airways allows for the simultaneous depiction of multiple contiguous airway segments Slice thickness can be altered to any dimension, but 3-7 mm generally gives adequate images. MPR images of the chest provide an excellent supplementary tool in the staging of lung cancer, particularly in delineating the relationship of the primary lesion to surrounding structures that are poorly appreciated on axial imaging. The best plane of imaging is usually tangential to the plane of interest (fig. 1) [20].

CHoOl et al. [20] demonstrated that the use of MPR images increases the confidence in diagnosing tumour invasion of anatomical structures, such as horizontal fissures and the diaphragm, that lie in the horizontal plane with coronal and sagittal imaging. The mean confidence for diagnosing general features for invasion and features for invasion the fissures and diaphragmatic invasion increased respectively from 1.68 to 2.08 , from 1.70 to 2.30 and from 1.20 to 2.00 for observer $A$ and from 1.50 to 1.80 , from 1.67 to 2.27 and from 0.71 to 1.14 for observer B. There was also a better inter-observer agreement in specifying the location of the primary lung cancer. HIGASHINO et al. [21] also confirmed in their study that the use of MPR images was useful for assessing the extent of regional tumours in non-small cell lung cancer patients.

Other reconstruction methods which are possible using $\mathrm{MDCT}$, are maximum intensity projection (MIP) images and 3-dimentional (3D) reconstructions. $\mathrm{CT}$ of the chest is the imaging technique with the highest sensitivity for the detection of pulmonary nodules. MIP images have the potential to reduce the number of overlooked small cancers (fig. 2). MIP projects the pixels with the highest attenuation values in a 2D format [22]. JANKOWSKI et al. [23] showed that MIP was the less time-consuming technique for the detection of pulmonary nodules. MIP also reduced the number of images to review by a factor of five compared with the series of 1-mm axial images. With regard to detection of nodules, the reader's sensitivities with MIP were greater than with $1-\mathrm{mm}$ images (33-45\% versus $22-47 \%$, respectively). KAwEL et al. [24] also 
showed that the use of thin slice MIP images was superior in the detection of pulmonary nodules. Sensitivity for pulmonary nodules was superior for 8-mm MIP and was significantly better than the sensitivities of all other tested techniques independent of nodule size and localisation [24].

3-D reconstructions include external and internal renderings [25]. External 3-D rendering of the airways is equivalent to $C T$ bronchography. 3-D segmentation of the tracheobronchial tree provides a rapid anatomical overview of the airways. 3-D reconstructions allow the recognition of mild and focal airway stenoses, providing accurate anatomically more relevant information on the shape, length and severity of airway stenoses. Internal 3-D rendering of the airways gives images equivalent to bronchoscopy. This virtual bronchoscopy (VB) is a CT-based imaging technique that allows a noninvasive intraluminal evaluation of the tracheobronchial tree. The principal parameter influencing the quality of the reconstruction remains the use of a thin slice thickness [26]. VB can be used as a noninvasive modality for identifying bronchial obstructions (fig. 3) and endoluminal lesions, as well as for assessing the tracheobronchial tree beyond stenoses [27]. FINKELSTEIN et al. [28] examined the potential role of VB and found that the sensitivity of VB was $100 \%$ for detection of obstructive lesions and $83 \%$ for endoluminal nonobstructive lesions, but the sensitivity for mucosal abnormalities was $0 \%$. The specificity of VB was $100 \%$. In mostcases, VB is able to depict direct tumour signs, such as a tumoural mass, a wall irregularity or a loss of cartilages. Indirect signs, such as stenosis or obstruction, compression or swelling, can often be visualised. However, mucosal infiltration, vascular dilatation and necrosis are usually missed [27]. VB can also be useful to visualise external compressions on the bronchial wall, not involving the mucosa (fig. 4). Correlating the position of the virtual bronchoscope with the axial $C T$ images usually allows the exact localisation and cause of the external compression to be defined [27].

Finally, MDCT may help reduce the radiation dose [29]. Low-dose CT (LDCT) for lung cancer screening in high-risk subjects is performed within clinical trials and has started to be used in routine clinical practice. Feasibility studies using low-dose $C T$ demonstrated a high proportion of non-small cell lung cancer at the initial examination with decreasing numbers of detected cancers at follow-up. [30, 31]. Menezes et al. [32] showed that LDCT can identify small lung cancers in an atrisk population. Sensitivity
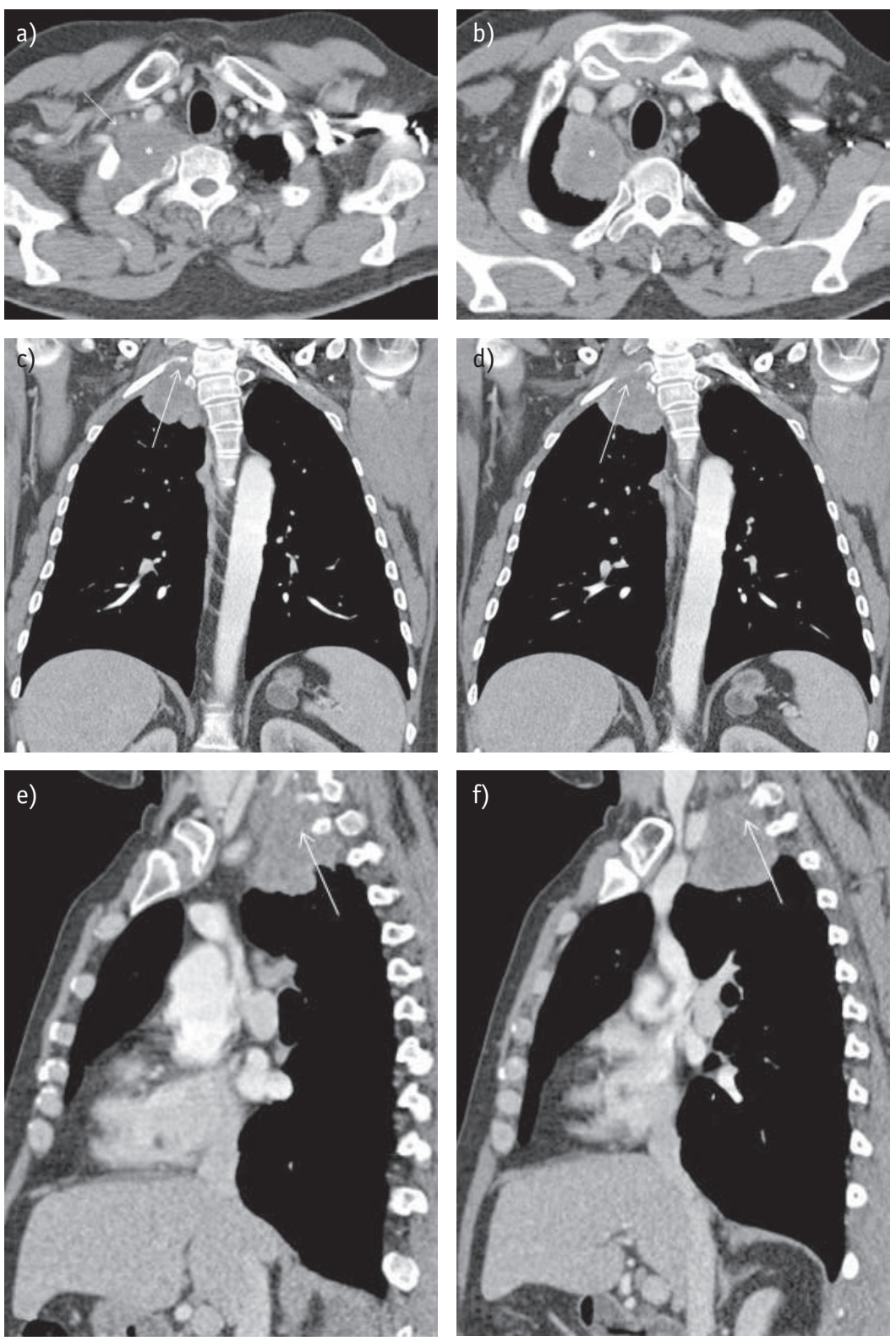

Figure 1

$a-b)$ Axial computed tomography images through the right upper lobe. A tumoural lesion is visible in the apex of the right upper lobe $\left(^{*}\right)$. This lesion, a pancoast tumour, cannot be delineated from the chest wall. Invasion in the chest wall (arrow) can be suspected but not clearly demonstrated. c-f) Two-dimensional multiplanar reconstruction (MPR) images in the $c-d)$ coronal and $e-f$ ) sagittal planes. Invasion of the chest wall can be clearly demonstrated in these MPR images (arrow).

and specificity of their protocol using LDCT, in successfully diagnosing early stage lung cancers were 87.7 and $99.3 \%$, respectively. Their diagnostic algorithm resulted in few false-positive invasive procedures.

\section{MR}

The lung remains a difficult organ for MR because of several limitations: the high susceptibility of MR to motion artifacts (i.e. pulsation and 

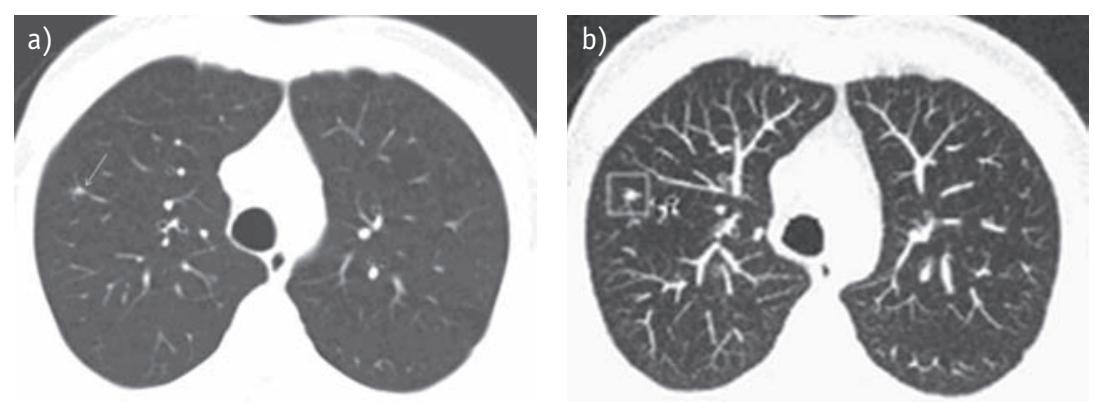

Figure 2

a) Axial, 5-mm slice thickness computed tomography (CT) image through the upper lobes at the level of the aortic arch. No clear lung nodules can be visualised at this axial CT image in the lung window. Only a slight irregular structure (arrow) can be retained in the right upper lobe, superimposed on a vascular structure. b) Maximum intensity projection (MIP) image through the upper lobes at the level of the aortic arch. A clear lung nodule can now be visualised (square). MIP images are better for indentifying small pulmonary nodules.

breathing), the intrinsic low proton density of lung parenchyma and the decrease in signal intensity due to air-soft tissue interfaces. This was the reason that MR was considered for many years as a useful problem-solving technique for specific instances when used in addition to $\mathrm{CT}$ [33]: 1) identification of tumour invasion in the chest wall and the mediastinal structures (pancoast tumour); 2) differentiation between solid and vascular hilar masses; 3) assessment of diaphragmatic abnormalities; and 4) the study and follow-up of mediastinal lymphoma. New applications, such as whole-body MR (WBMR) imaging, may significantly increase MR-sensitivity in the near future. WBMR has shown advantages for the detection of distant metastatic disease [34]. Diffusion weighted imaging (DWI) is a
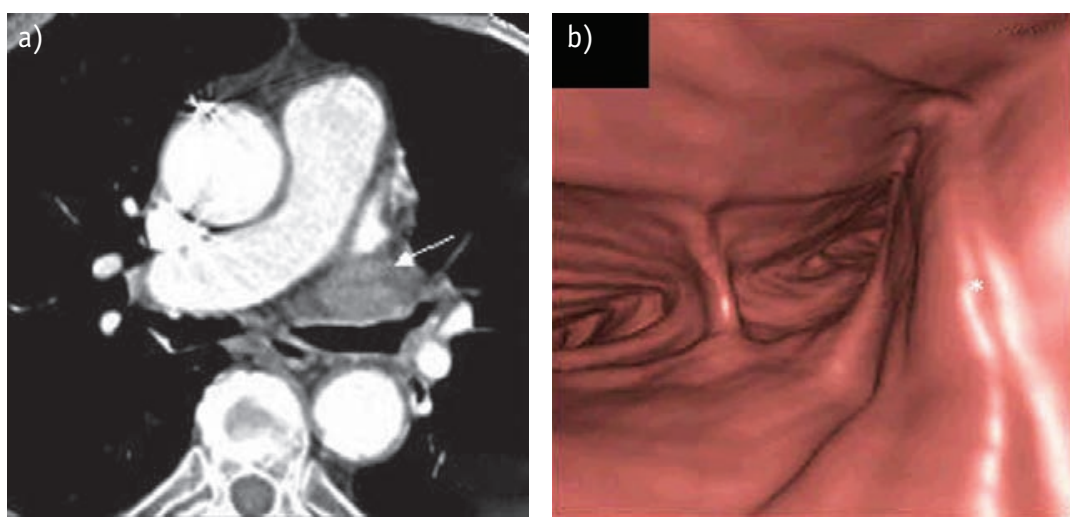

Figure 4

Axial computed tomography (CT) image and virtual bronchoscopic view. a) The axial CT image shows a nodular lesion ventral of the left main stem bronchus. This lesion corresponded with an elarged lymph node. This lymph node showed an impression on the anterior wall of the left main stem bronchus. b) The bronchoscopic view demonstrated this impression, which was visible on the anterior wall of the bronchus $\left(^{*}\right)$.
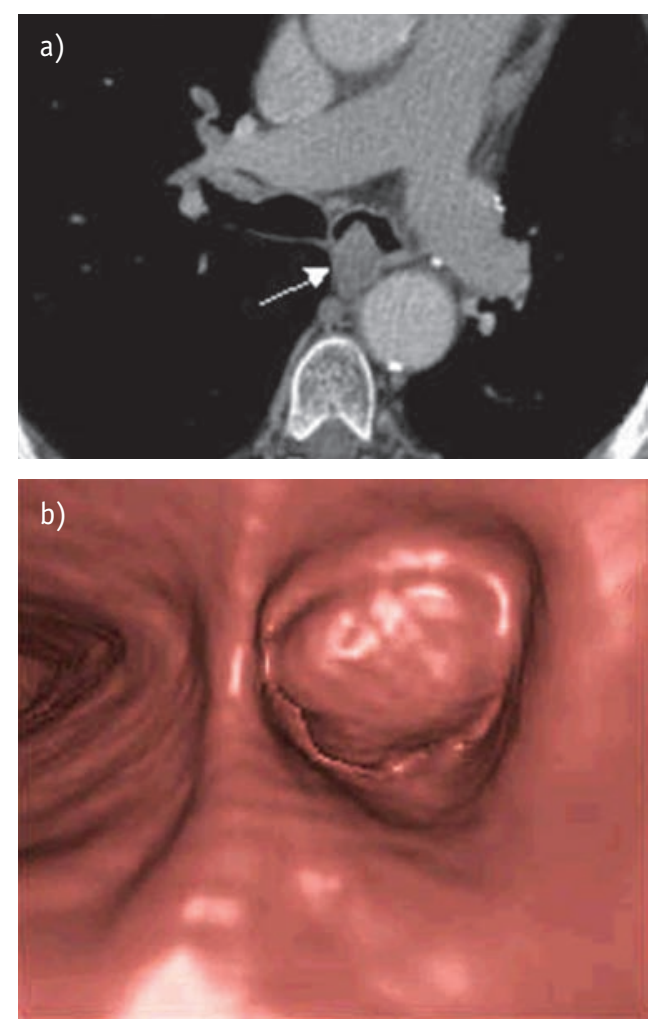

Figure 3

a) Axial computed tomography (CT) images at the level of the carina. This image shows a nodular lesion in the posterior wall of the left main stem bronchus, which was suspected to be a tumoural lesion. This was confirmed histopahologically. b) Virtual bronchoscopic view of the carina. In the left main stem bronchus, a large polypoid lesion can be visualised, corresponding with the nodular lesion visible on CT scan. With virtual bronchoscopy, this lesion can be passed to evaluate the bronchial tree distal of this tumoral lesion.

powerful imaging tool that provides unique information related to tumour cellularity and the integrity of the cellular membrane. The DWI sequence is made susceptible to the differences in water mobility. The motion of water molecules is more restricted in tissues with a high cellular density associated with numerous intact cell membranes (e.g. tumour tissue). This technique can be applied for tumour detection and tumour characterisation and for the monitoring of response to treatment (figs 5-8) $[35,36]$.

\section{Positron emission tomography and integrated PET/CT}

Positron emission tomography (PET) is a sensitive and specific imaging technique that allows in vivo imaging of metabolic pathways in human tissue [37]. For this purpose, PET uses radioisotopes of natural elements. In most oncological 

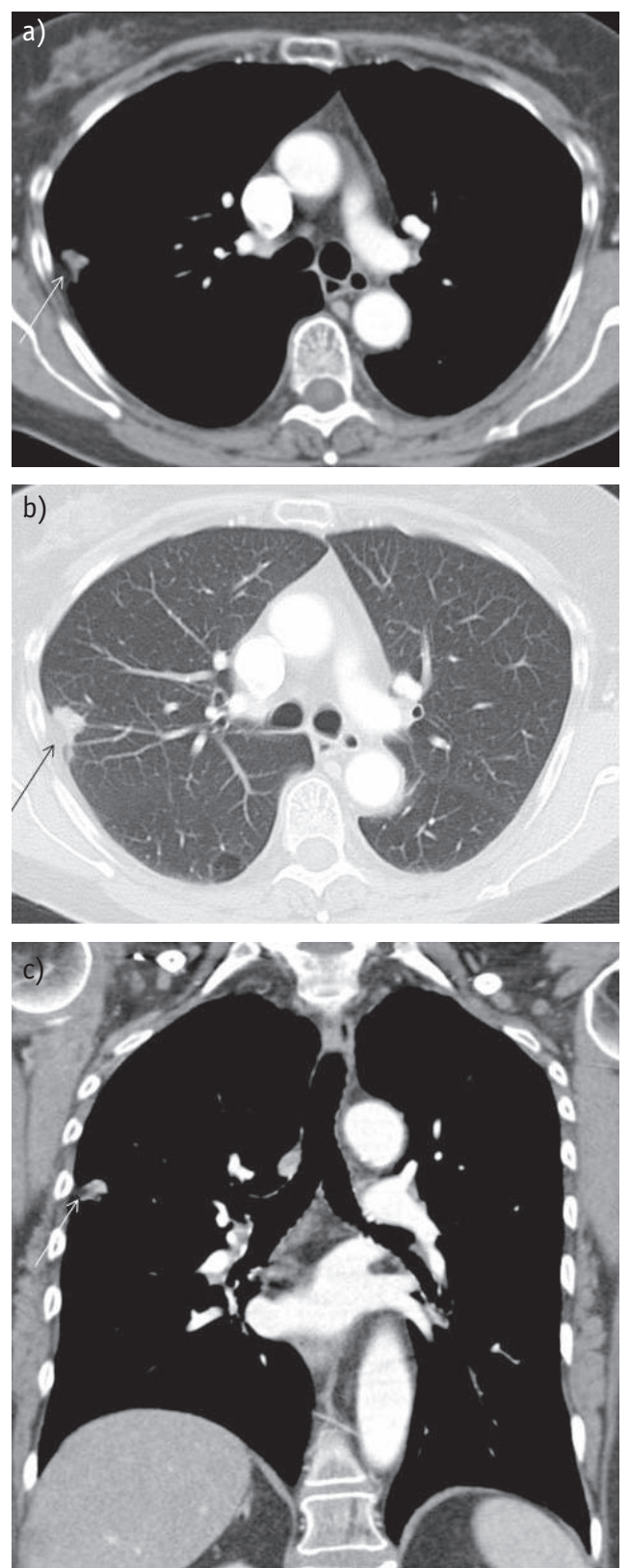

Figure 5

Incidental finding of a nodular lesion in the right upper lobe. a) Axial mediastinal window, b) axial lung window and c) coronal multiplanar reconstruction images showed a nodular lesion in the right upper lobe with a pleural tail (arrow). This lesion was suspected to be tumoural. However, fluoro-deoxy-glucose (FDG) positron emission tomography examination did not show FDG uptake in this lesion.

imaging, such as thoracic oncology, fluoro-deoxyglucose (FDG) is used as radioisotope. FDG is a D-glucose molecule in which an hydroxyl group in the 2- position is replaced by a positronemitting isotope of fluorine $\left({ }^{18} \mathrm{~F}\right)$. FDG is taken up and metabolised by cells in the same fashion as glucose but once FDG is phosphorylated, it is
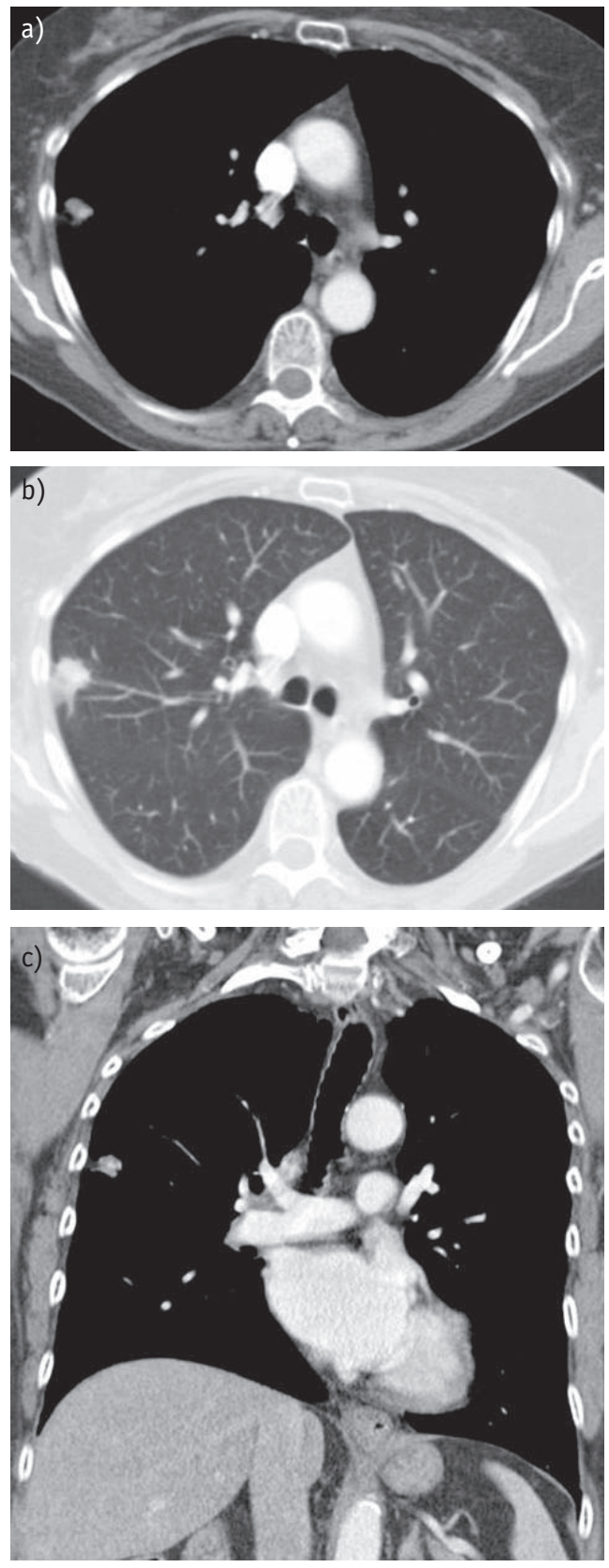

Figure 6

Control computed tomography of the nodular lesion in the right upper lobe after 3 months. a) Axial mediastinal window, b) axial lung window and c) coronal multiplanar reconstruction images showed the same lesion. The volume of the lesion was slightly increased.

not further metabolised, but rather becomes trapped intracellularly [38]. Malignant cells have higher rates of glucose metabolism than normal cells, and therefore accumulate greater amounts of the radiolabeled FDG. Radioisotopes like ${ }^{18} \mathrm{~F}$ or ${ }^{11} \mathrm{C}$ allow the synthesis of numerous positronemitting radiopharmaceuticals. The biodistribution of the positronemitting tracers is measured 

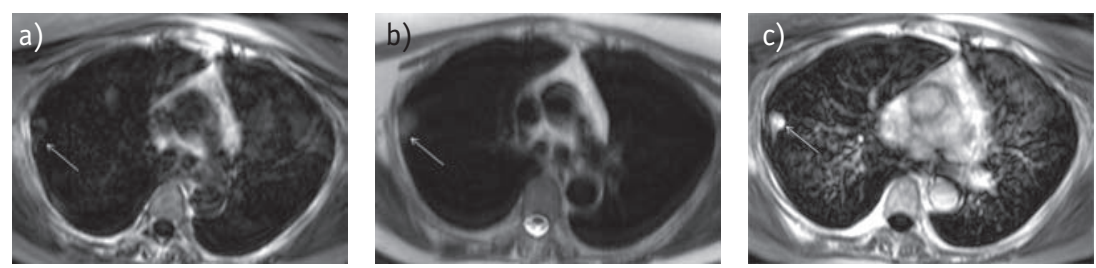

Figure 7

Axial magnetic resonance (MR) imaging. a) T1 and b) T2 MR weighted images demonstrated a nodular lesion in the right upper lobe (arrow). c) There was contrast captation in this lesion on the T weighted MR imaging after i.v. contrast administration (arrow).

using a PET camera. A positron transverses a short distance through the tissue until it combines with an electron in the surrounding media (annihilation). This generates a pair of photons which travel in nearly opposite directions ( $180^{\circ}$ apart) with an energy of $511 \mathrm{keV}$ each. These opposite photons can be detected by detector pairs installed in a ring shaped pattern in the PET camera. Photons that simultaneously interact with these detectors are registered as decay events. Based on these registrations, tomographic images of the regional radioactivity distribution are reconstructed (emission images). When quantitative assessment of
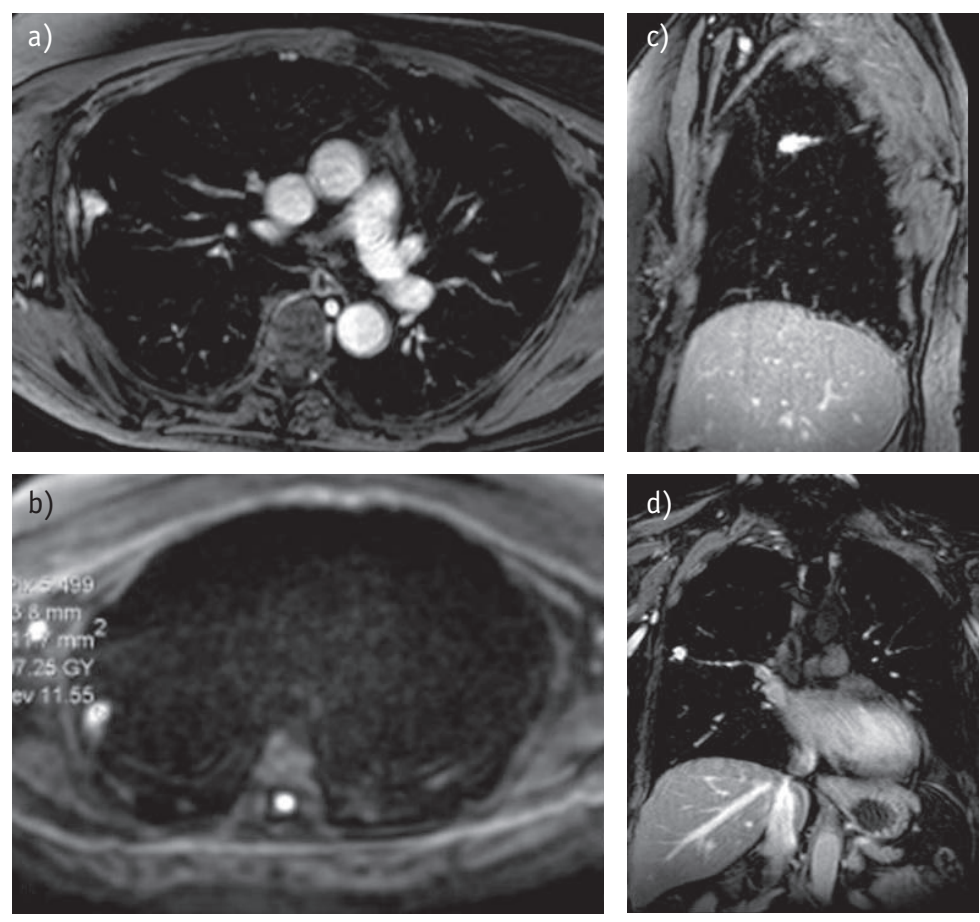

Figure 8

Diffusion weighted magnetic resonance imaging. $a-b)$ Axial, c) sagittal and d) coronal diffusion weighted magnetic resonance imaging showed a very intense nodular structure in the right upper lobe, suspected to be a tumoural lesion. The lesion was removed surgically and adenocarcinoma was confirmed on histopathology.
FDG metabolism is needed, e.g. for the assessment of the metabolic response to an antineoplastic treatment, correction for soft-tissue attenuation is crucial. To achieve this attenuation correction, a set of corresponding images (transmission scan) is acquired with an external highenergy photon source (Germanium-68 or Cesium-137). This transmission scan adds at least $50 \%$ to the scanning time and results in data with a relatively high noise level [39]. This transmission scan can be performed prior to injection of the tracer (cold transmission) or afterwards (hot transmission).

The most widely used semi-quantitative index of FDG uptake is the standardised uptake value (SUV). To calculate the SUV, the measured tumour radiotracer concentration $(\mathrm{Q})$ is normalised to the injected activity (Qinj) and to the body weight (W) of the patient: SUV $=(Q \times W) /$ Qinj. Lung lesions showing up as "hot spots" on the scan, with a SUV of $>2.5$ have a high likelihood of being malignant [40]. FDG-PET has proven useful in: 1) diagnosing and staging lung cancer; 2) monitoring the efficacy of treatment; and 3) defining the biological target volume for radiation treatment planning $[41,42]$

$\mathrm{PET} / \mathrm{CT}$ is a combined anatomo-metabolic imaging technique. The first integrated PET/CT machine came into clinical practice in 1998, and it is now inevitable that all future installations of PET machines will be in the form of PET/CT. Integrated PET/CT is the combination of two different imaging techniques in one machine: $C T$ giving anatomical information and PET giving metabolic information. The additional information gained with integrated PET/CT can be: 1) detection of lesions initially not seen on $C T$ or PET; 2) more precise localisation of lesions; 3) better delineation of lesions from their surrounding structures; and 4) better characterisation of lesions as benign or malignant [41, 43]. PET/CT is the best noninvasive imaging technique for the correct prediction of T-stage. DE WEVER et al. [44] showed that PET/CT correctly predicted the $T$ (tumour) stage in $82 \%$ of cases, in comparison with 55,68 and $76 \%$ of cases when PET, CT and visual correlation of PET and $C T$ were used, respectively. However, all imaging methods can over- and under-stage the tumour. One of the most important attributes of PET/CT is the ability to distinguish between tumour and distal atelectasis (fig. 9). Initial studies for $\mathrm{N}$ (lymph node) staging demonstrated a pooled average sensitivity, specificity, positive predictive value, negative predictive value and accuracy of PET/CT of 73, 80, 78, 91 and 87\%, respectively 
[44]. The advantage of integrated PET/CT imaging in $\mathrm{M}$ (metastases) staging is the ability to exactly locate a focal abnormality on PET images. PET/CT was found to be the best noninvasive imaging technique in evaluating distant metastases in several studies [45]. Another advantage, more specifically related to PET is that, since the CT data can be used for the attenuation correction of the PET images, the examination time of this PET examination may be reduced by up to $30 \%$ [46].

\section{Conclusion}

State-ofthe-art spiral and multi-detector CT scanners are able to present very detailed 2D or even $3 \mathrm{D}$ images of the tumour and its extent in the surrounding tissues. However, despite this improved image quality, there are still a lot of cases where CT may leave this in doubt. MR may be used as a problem solving modality. The role of CT in determining nodal involvement is limited but important. It offers the surgeon a road-map of the lymph nodes in the hilum and the mediastinum and guides him towards the nodes that need a biopsy. Combining CT and PET will certainly improve nodal staging of the mediastinum and will reduce the number of unnecessary interventional procedures. Integrated PET/CT has proven to be the best imaging technique for staging patients with lung cancer. It combines morphological and
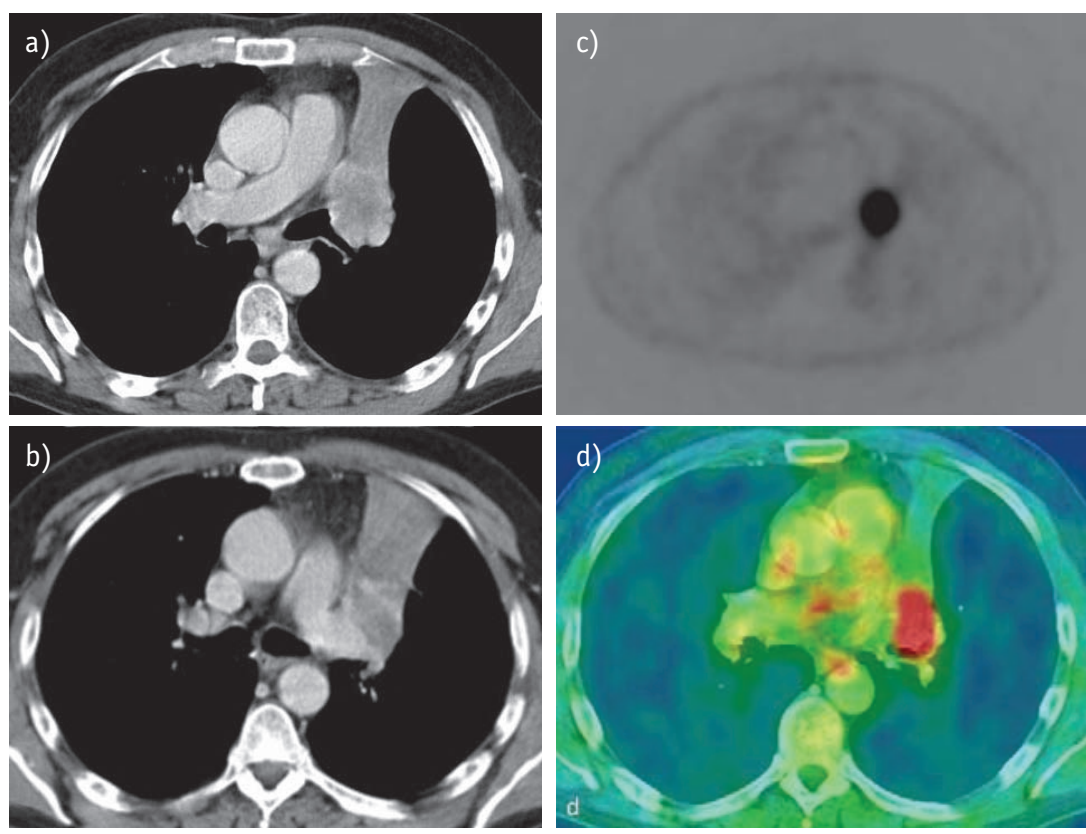

Figure 9

Integrated positron emission tomography (PET)/computed tomography (CT). a-b) Axial CT images showed a central tumour in the left lung with a retro-obstructive atelectasis of the left upper lobe. c) PET showed a hotspot in the left lung corresponding with the central tumour. No fluoro-deoxy-glucose uptake was visible in the retro-obstructive atelectasis. Integrated PET/CT imaging showed the hotspot located on the central tumour. With integrated PET/CT, a better discrimination between tumour and retro-obstructive atelectasis can be demonstrated.

metabolic information. With the development of new MR acquisitions MR becomes a wellpromised tool in the future of staging lung cancer patients.

\section{References}

1. Schaefer-Prokop C, Prokop M. New imaging techniques in the treatment guidelines for lung cancer. Eur Respir J 2002; 19: Suppl. 35, 71s-83s.

2. Zinn B, Monroe J. The lordotic position in fluoroscopy and roentgenography of the chest. Am J Roentgenol Radium Ther Nucl Med 1956; 75: 682-700.

3. McAdams HP, Samei E, Dobbins J 3rd, et al. Recent advances in chest radiography. Radiology 2006; 241: 663-683.

4. Schaefer-Prokop C, Neitzel U, Venema HW, et al. Digital chest radiography: an update on modern technology, dose containment and control of image quality. Eur Radiol 2008; 18: 1818-1830.

5. Schaefer-Prokop C, Uffmann M, Eisenhuber E, et al. Digital radiography of the chest: detector techniques and performance parameters. J Thorac Imaging 2003; 18: 124-137.

6. MacMahon H, Doi K. Digital chest radiography. Clin Chest Med 1991; 12: 19-32.

7. Nitrosi A, Borasi G, Nicoli $F$, et al. A filmless radiology department in a full digital regional hospital: quantitative evaluation of the increased quality and efficiency. J Digit Imaging 2007; 20: 140-148.

8. Kelcz F, Zink FE, Peppler WW, et al. Conventional chest radiography versus dual-energy computed radiography in the detection and characterization of pulmonary nodules. Am J Roentgenol 1994; 162: 271-278.

9. Li F, Engelmann R, Doi K, et al. Improved detection of small lung cancers with dual-energy subtraction chest radiography. Am J Roentgenol 2008; 190: 886-891.

10. MacMahon $\mathrm{H}$, Li F, Engelmann R, et al. Dual-energy subtraction and temporal subtraction chest radiography. J Thorac Imaging 2008; 23: 77-85.

11. Kido $\mathrm{S}$, Ikezoe J, Naito $\mathrm{H}$, et al. Clinical evaluation of pulmonary nodules with single-exposure dual-energy subtraction chest radiography with an iterative noise-reduction algorithm. Radiology 1995; 194: 407-412.

12. Brett GZ. Earlier diagnosis and survival in lung cancer. Br Med J 1969; 4: 260-262.

13. Fontana RS, Sanderson DR, Woolner LB, et al. Lung cancer screening: the Mayo program. J Occup Med 1986; 28: 746-750.

14. Spratt JS Jr, Spjut HJ, Roper CL. The frequency distribution of the growth rates and the estimated duration of primary pulmonary carcinomas. Acta Unio Int Contra Cancrum 1963; 19: 1270-1275. 
15. MacDonald SL, Hansell DM. Staging of non-small cell lung cancer: imaging of intrathoracic disease. Eur J Radiol 2003; 45: 18-30.

16. Kalra MK, Maher MM, D'Souza R, et al. Multidetector computed tomography technology: current status and emerging developments. J Comput Assist Tomogr 2004; 28: Suppl. 1, S2-S6.

17. Flohr TG, Schaller S, Stierstorfer K, et al. Multi-detector row CT systems and image-reconstruction techniques. Radiology 2005; 235: 756-773.

18. Honda 0, Johkoh T, Yamamoto S, et al. Comparison of quality of multiplanar reconstructions and direct coronal multidetector CT scans of the lung. Am J Roentgenol 2002; 179: 875-879.

19. Prokop M. General principles of MDCT. Eur J Radiol 2003; 45: Suppl. 1, S4-S10.

20. Chooi WK, Matthews S, Bull MJ, et al. Multislice computed tomography in staging lung cancer: the role of multiplanar image reconstruction. J Comput Assist Tomogr 2005; 29: 357-360.

21. Higashino T, Ohno Y, Takenaka D, et al. Thin-section multiplanar reformats from multidetector-row CT data: utility for assessment of regional tumor extent in non-small cell lung cancer. Eur J Radiol 2005; 56: 48-55.

22. Peloschek P, Sailer J, Weber M, et al. Pulmonary nodules: sensitivity of maximum intensity projection versus that of volume rendering of 3D multidetector CT data. Radiology 2007; 243: 561-569.

23. Jankowski A, Martinelli T, Timsit JF, et al. Pulmonary nodule detection on MDCT images: evaluation of diagnostic performance using thin axial images, maximum intensity projections, and computer-assisted detection. Eur Radiol 2007; 17: 3148-3156.

24. Kawel N, Seifert B, Luetolf M, et al. Effect of slab thickness on the CT detection of pulmonary nodules: use of sliding thin-slab maximum intensity projection and volume rendering. Am J Roentgenol 2009; 192: 1324-1329.

25. De Wever W, Bogaert J, Verschakelen JA. Virtual bronchoscopy: accuracy and usefulness-an overview. Semin Ultrasound CT MR 2005; 26: 364-373.

26. Rogalla P, Ruckert JC, Schmidt B, et al. [Virtual bronchoscopy.]. Radiologe 2001; 41: 261-268.

27. De Wever W, Vandecaveye V, Lanciotti S, et al. Multidetector CT-generated virtual bronchoscopy: an illustrated review of the potential clinical indications. Eur Respir J 2004; 23: 776-782.

28. Finkelstein SE, Summers RM, Nguyen DM, et al. Virtual bronchoscopy for evaluation of malignant tumors of the thorax. J Thorac Cardiovasc Surg 2002; 123: 967-972.

29. Kusumoto M, Tateishi U, Arai Y, et al. [Diagnostic imaging of lung cancer on multislice CT (MDCT).]. Gan To Kagaku Ryoho 2005; 32: 759-764.

30. Bellomi M, Rampinelli C, De Fiori E, et al. Lung cancer screening update.. Cancer Imaging 2009; 9: Spec No A, S122S125.

31. Diederich S, Wormanns D. Impact of low-dose CT on lung cancer screening. Lung Cancer 2004; 45: Suppl. 2, S13S19.

32. Menezes RJ, Roberts HC, Paul NS, et al. Lung cancer screening using low-dose computed tomography in at-risk individuals: the Toronto experience. Lung Cancer; 67: 177-183.

33. Muller NL. Computed tomography and magnetic resonance imaging: past, present and future. Eur Respir J 2002; 19: Suppl. 35, 3s-12s.

34. Schmidt GP, Reiser MF, Baur-Melnyk A. Whole-body MRI for the staging and follow-up of patients with metastasis. Eur J Radiol 2009; 70: 393-400.

35. Matoba M, Tonami H, Kondou T, et al. Lung carcinoma: diffusion-weighted mr imaging-preliminary evaluation with apparent diffusion coefficient. Radiology 2007; 243: 570-577.

36. Koh DM, Collins DJ. Diffusion-weighted MRI in the body: applications and challenges in oncology. AJR Am J Roentgenol 2007; 188: 1622-1635.

37. Jones T. The imaging science of positron emission tomography. Eur J Nucl Med 1996; 23: 807-813.

38. Pauwels EK, McCready VR, Stoot JH, et al. The mechanism of accumulation of tumour-localising radiopharmaceuticals. Eur J Nucl Med 1998; 25: 277-305.

39. Burger C, Goerres G, Schoenes S, et al. PET attenuation coefficients from CT images: experimental evaluation of the transformation of CT into PET 511-keV attenuation coefficients. Eur J Nucl Med Mol Imaging 2002; 29: 922-927.

40. Sihoe AD, Yim AP. Lung cancer staging. J Surg Res 2004; 117: 92-106.

41. De Wever W, Stroobants S, Verschakelen JA. Integrated PET/CT in lung cancer imaging: history and technical aspects. JBR-BTR 2007; 90: 112-119.

42. de Geus-Oei LF, van der Heijden HF, Corstens FH, et al. Predictive and prognostic value of FDG-PET in nonsmall-cell lung cancer: a systematic review. Cancer 2007; 110: 1654-1664.

43. Bar-Shalom R, Yefremov N, Guralnik L, et al. Clinical performance of PET/CT in evaluation of cancer: additional value for diagnostic imaging and patient management. J Nucl Med 2003; 44: 1200-1209.

44. De Wever W, Ceyssens S, Mortelmans L, et al. Additional value of PET-CT in the staging of lung cancer: comparison with CT alone, PET alone and visual correlation of PET and CT. Eur Radiol 2007; 17: 23-32.

45. De Wever W, Stroobants S, Coolen J, et al. Integrated PET/CT in the staging of nonsmall cell lung cancer: technical aspects and clinical integration. Eur Respir J 2009; 33: 201-212.

46. Antoch G, Stattaus J, Nemat AT, et al. Non-small cell lung cancer: dual-modality PET/CT in preoperative staging. Radiology 2003; 229: 526-533. 